\title{
Inferring Human Activity using Overcomplete Dictionary Based Pattern Recognition
}

\author{
Wei-Chuan Lin $^{1} \quad$ Wei Li ${ }^{1,2} \quad$ Winston K.G. Seah ${ }^{1}$ \\ ${ }^{1}$ School of Engineering and Computer Science \\ Victoria University of Wellington, PO Box 600 \\ Wellington 6140, New Zealand \\ Email: \{wei-chuan.lin,winston.seah\}@ecs.vuw.ac.nz
}

\author{
T. Aaron Gulliver ${ }^{2}$ \\ ${ }^{2}$ Department of Electrical and Computer Engineering \\ University of Victoria \\ Victoria, Canada V3W 3P6 \\ Email: \{wli, agullive $\}$ ece.uvic.ca
}

\begin{abstract}
Inferring human activity is often achieved using specialized sensors and beacons such as accelerometers, pedometers and motion sensors on wireless nodes. These sensor nodes compose a Wireless Sensor Network in a coordinated way. However, these sensors are too expensive for large deployment, which affects performance. In addition, the transmission accuracy of wireless communications suffers from radio irregularities caused by obstacles such as human bodies in the environment. The human body selectively reflects, diffracts and scatters the radio signal which affects the received signal strength. This paper presents an approach to detecting human activity using fluctuations in the received signal strength. These fluctuations are detected using an overcomplete dictionary based pattern recognition algorithm. Performance results are presented which show that the proposed system has an accuracy of $86 \%$ in detecting human activity. Moreover, the detection algorithm can be implemented in software without modifying the existing infrastructure. As result, this is a promising technology for security and surveillance applications.
\end{abstract}

Index Terms-Wireless Sensor Network; intrusion detection; inferring human activity

\section{INTRODUCTION}

A Wireless Sensor Network (WSN) consists of distributed wireless sensor nodes that operate in a coordinated way to monitor physical or environmental conditions [1]. Each of these nodes, often referred to as "motes", has processing and wireless transmission capabilities, although these tend to be limited. In addition, the wireless nodes contain specialised sensors which are able to monitor a variety of ambient condition such as temperature, pressure and humidity. The features of WSNs such as low-cost and low-power with multifunctional sensing capabilities [1] have opened up new applications in many areas such as health, military, and security.

Motes typically have resource constraints such as finite on-board battery power, limited communications bandwidth, and limited memory [2]. However, problems such as limited battery capacity can be alleviated through the deployment of a large number of wireless sensors for redundancy. A problem which cannot easily be resolved is radio irregularity [3]. Radio irregularity is determined by the device and the media [2]. Device properties include antenna parameters, transmission power, and receiver sensitivity, while media properties include background noise and obstacles in the propagation channel.
Two components of signal propagation considered to be key causes of radio irregularity are path loss and transmission power [2]. The radio signal may be reflected, diffracted and/or scattered in the channel [4]. The impact of radio irregularity depends on the obstacles and the wireless signals transmitted. The human body selectively reflects, diffracts, and scatters radio signals such that the radio irregularity varies over time. Consequently, the received signal strength at the receiver fluctuates. It has been observed [3] that there are certain fluctuation patterns associated with the presence of human activity. By considering these patterns, an overcomplete dictionary based pattern recognition technique [5] is presented in this paper which can improve activity detection accuracy.

In the next section, we briefly discuss related work on motion detection using receiving signal strength in WSNs. In Section III, we describe the characteristics of wireless signal strength in the presence of human activity. A novel detection algorithm using overcomplete dictionary based pattern recognition for inferring human activity is proposed in Section IV. The detection performance is evaluated in Section V, and some conclusions are given in Section VI.

\section{RELATED WORK}

Precise motion inference can be achieved by specialised sensors and beacons such as accelerometers, pedometers and motion sensors [6]. However, large deployment of these devices is impractical due to their high cost and obtrusive characteristics. This has motivated the development of alternative motion detection techniques suitable for current network infrastructures without hardware alterations. Although small-scale fading and shadowing can affect the received signal strength, it should be stable while the nodes are static. In [7], the characteristics of the received signal strength were exploited for various applications and validated using the Mica2 mote by Crossbow.

Wireless sensing is also possible using the existing wireless infrastructure such as WiFi and GSM. In [8], a novel motion detection algorithm was proposed called Spectrally Spread Motion Detection (SpecSMD). This algorithm is able to classify user motion as either 'moving' or 'still' by examining the characteristics of the Received Signal Strength Indicator (RSSI) of a WiFi signal. Users must therefore carry a WiFi 
device. The algorithm was shown to achieve an accuracy of $94 \%$. A neural network approach has also been used to distinguish user motion using fluctuations in a GSM signal [9].

In this paper, we propose motion detection by distinguishing the RSSI pattern using an overcomplete dictionary based pattern recognition algorithm. This algorithm is a software solution which can be used with existing wireless communication devices for intrusion detection without employing additional hardware.

\section{Characteristics OF THE WIRELESS SignAL STRENGTH}

Wireless signals may be reflected, diffracted and/or scattered during transmission. According to [3], there are certain patterns associated with the existence of a moving object in an environment. Two Crossbow ${ }^{\circledR}$ TelosB motes were placed $4 \mathrm{~m}$ apart at a height of $1.5 \mathrm{~m}$. One mote transmitted packets to the other mote with a 0.25 second inter-packet time interval. The RSSI fluctuation patterns were found to be consistent regardless of the environmental factors or absolute RSSI values [3]. For a given packet $p_{i}$, the RSSI fluctuation were calculated as $F\left(p_{i}\right)=S\left(p_{i}\right)-S\left(p_{i-1}\right)$. For example, the sequence of RSSI values $1,2,4,5,8,7,6$ produces the RSSI fluctuation values $0,+1,+2,+1,+3,-1,-1$. The study reported consistent but different patterns of RSSI fluctuations with and without movement. An example of these results is shown in Fig. 1. The RSSI values have less fluctuation when there is no movement in the environment, and a wider distribution in the presence of human movement.

\section{INFERRING HUMAN ACTIVITY}

In this section, we present a movement detection system using an overcomplete dictionary based pattern recognition algorithm. This detection algorithm has been employed in ultra-wideband communications systems [5]. A flowchart of the proposed system is shown in Fig. 2(a). It begins with the measured RSSI readings and calculates the frequency of RSSI fluctuations over a window of $N$ packets. The overcomplete dictionary based pattern recognition algorithm takes this frequency data as input. The pattern recognition algorithm is shown in Fig. 2(b).

The overcompleted dictionary $\mathbf{D}_{N}$ consists of two matrices

$$
\mathbf{D}_{N}=\left[\begin{array}{ll}
\mathbf{I} & \mathbf{H}
\end{array}\right],
$$

where

I is the spike-like dictionary with size of $16 \times 16$, and

$\mathbf{H}$ is the Walsh noise dictionary with size of $16 \times 16$.

The algorithm decomposes the RSSI frequency data vector $\mathrm{y}$ with dictionary $\mathbf{D}_{N}$ using $l_{1}$ norm minimisation [10][11] to obtain the solution

$$
\mathbf{x}=\left[\begin{array}{l}
\gamma_{\mathrm{I}} \\
\gamma_{\mathrm{H}}
\end{array}\right]
$$

where

$$
\mathbf{y}=\mathbf{D}_{N} \mathbf{x}=\mathbf{I} \gamma_{\boldsymbol{I}}+\mathbf{H} \gamma_{\mathbf{H}},
$$

The RSSI fluctuations patterns shown in Fig. 1 were decomposed with dictionary $D_{N}$ using $l_{1}$ norm minimisation. The results with and without movement are shown in the Fig. 3 . Atom indices 1 to 16 correspond to the spike-like dictionary and 17 to 32 to the Walsh dictionary. Fig. 3 clearly shows that the maximum coefficient lies between atom 1 to 16 when there is no movement, and between 17 to 32 when movement occurs. movement pattern is decomposed. Thus the decision rule is no movement when the largest peak is located among atoms 1 to 16 , and movement otherwise.
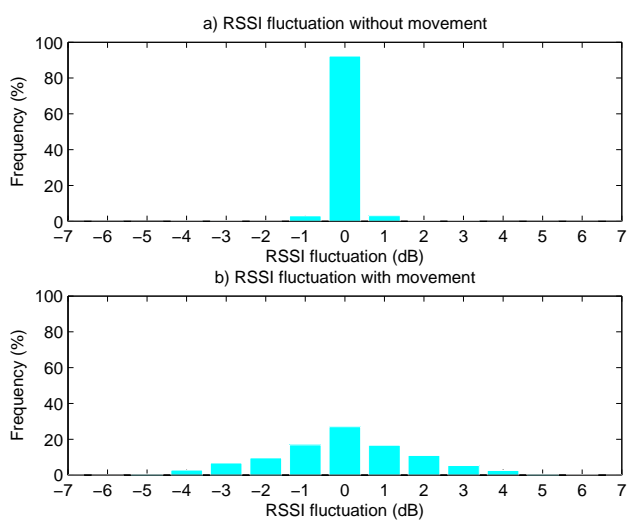

Fig. 1. RSSI fluctuation patterns

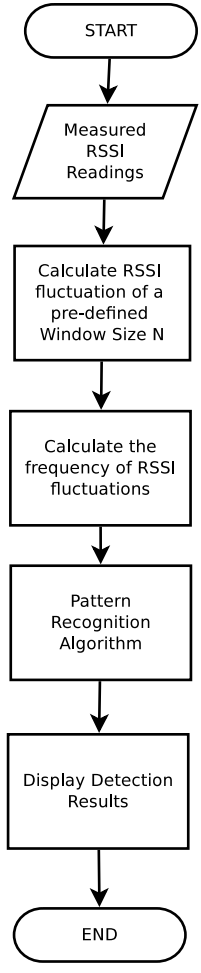

(a)

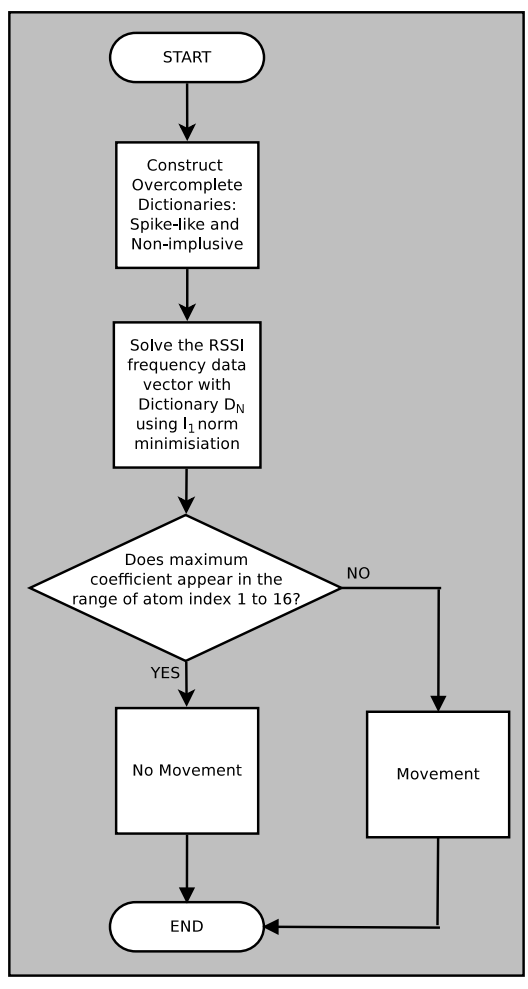

(b)
Fig. 2. (a) Flowchart of the proposed movement detection system. (b) The overcomplete dictionary based pattern recognition 


\section{Detection Performance Evaluation}

In [3], a series of experiments was performed in different locations to evaluate their proposed algorithm. The experiments were conducted in three different meeting rooms of approximate area $6 \mathrm{~m} \times 4 \mathrm{~m}$. The motes were placed at a height of $1.5 \mathrm{~m}$ and spaced $4 \mathrm{~m}$ apart. Over a 20 minute period, a person walked between the motes every two minutes. RSSI samples were obtained for every received packet. Therefore, the sampling rate is the inter-packet interval of $0.25 \mathrm{sec}$, so that 100 samples implies a duration of $25 \mathrm{sec}$.

\section{A. Detection results}

The data from [3] was used with the proposed detection algorithm. The results using the data collected in Room 1 with window size $N=100$ samples are shown in Fig. 4. The proposed algorithm was able to detect every movement that occurred in the environment, but the accuracy of the movement duration was low. Note that there were no false positive detections. The inaccuracy in the inferred movement durations is due in part to the window size. Therefore, the algorithm was repeated with the window size reduced to $N=50$ samples. The results given in Fig. 5 show that reducing the window size improves the accuracy of both the inferred movement durations and detection latency. Considering that the RSSI fluctuation statistics were gathered for a window of size 100 samples, the pattern of the resulting statistics is not obvious due to this large window size. Non-movement and movement patterns might be mixed over a large window size. For instance, there is a missed detection at sample index of 1300 when $\mathrm{N}$ is set to 100 samples. However, the detection result is correct when the window size is reduced to 50 samples. With $\mathrm{N}=100$ samples, the frequency of RSSI fluctuation is calculated with samples from 1201 to 1300 where 1201 to 1229 has non-movement. Therefore, the RSSI fluctuations have a small spike between -1 to 1 as shown in Fig. 6. This results in an incorrect detection outcome. A smaller window size can improved this performance both timing and detection duration.

\section{B. Optimal Window Size}

The effect of different window sizes on the detection error is now considered. We aim to find an optimal detection window size that minimise the detection error. The detection algorithm was repeated with window sizes from $N=10$ to 150 samples. The detection error rate was calculated by dividing the number of differences between the actual and detected movement results, by the total number of samples. Fig. 7 shows that the detection error rate for Room 1 is lowest when the window size is $N=40$ samples for Room 1 . The detection error rate is high when the window size is small because there are too few samples to effectively detect the pattern. As the window size increases beyond 40 samples, the detection error rate increases due to the reason discussed previously. The detection algorithm can produce detection error due to the mixture of movement and non-movement patterns within a large window size. The detection results for Rooms 2 and 3 are shown in
Fig. 8 and 9. The best window sizes are 40 and 55 samples for Rooms 2 and 3, respectively. However, the overall error rate of Room 2 is extremely high compared to the results of Room 1 and 3. This issue needs to be further studied by performing more experiments. We observed that a window size of 45 samples is suitable for every environment. The detection results of Room 1 and 3 are shown in Fig. 10 to 12 with window size equals to 45 samples. The detection algorithm achieves an overall accuracy of $86 \%$ with no false positive detections.

\section{CONCLUSIONS}

In this paper, the feasibility of using wireless sensor motes for motion detection has been demonstrated. It was shown that the absolute received signal strength varies while the fluctuations remain stable regardless of the environment. These fluctuations can be used to detect human motion in the wireless environment. It was also shown that the detection accuracy can be improved by using an overcomplete dictionary based pattern recognition algorithm. An $86 \%$ detection rate was achieved with no false positive detections. This approach to human activity detection can be implemented with standard communication equipment to provide intrusion detection. If the detection information obtained is insufficient, the proposed algorithm can be used in conjunction with a security system. For example, it can be employed to turn on surveillance cameras when a moving object is detected in a restricted area. This is a promising technology for intrusion detection because it does not need additional hardware, and thus can be considered as an option for future security applications.

\section{REFERENCES}

[1] I. F. Akyildiz, W. Su, Y. Sankarasubramaniam, and E. Cayirci, "Wireless sensor networks: A survey," Computer Networks, vol. 38, no. 4, pp. 393422, Mar. 2002.

[2] I. Onat and A. Miri, "An intrusion detection system for wireless sensor networks," in Proc. IEEE Int. Conf. on Wireless And Mobile Computing, Networking And Commun. (WiCOM), Aug. 2005, pp. 253-259.

[3] P. Lee, W. Seah, H.-P. Tan, and Z. Yao, "Wireless sensing without sensors - An experimental approach," in Proc. IEEE Int. Symp. on Personal, Indoor and Mobile Radio Communications (PIMRC), Sept. 2009, pp. 62-66.

[4] P. Shankar, Introduction to Wireless Systems, New York: Wiley, 2001.

[5] W. Li and T. A. Gulliver, "Overcomplete dictionary based ultrawideband signal detection," in Proc. IEEE Wireless Commun. and Networking Conf., Apr. 2006, pp. 878-883.

[6] C. Randell and H. H. Muller, "Context awareness by analysing accelerometer data," in Proc. IEEE Int. Symp. on Wearable Computers (ISWC), Oct. 2000, pp. 175-176.

[7] K. Woyach, D. Puccinelli, and M. Haenggi, "Sensorless sensing in wireless networks: Implementation and measurements," in Proc. IEEE Int. Workshop on Modeling and Optimization in Mobile, Ad Hoc and Wireless Networks, Apr. 2006, pp. 1-8.

[8] K. Muthukrishnan, M. Lijding, N. Meratnia, and P. Havinga, "Sensing motion using spectral and spatial analysis of WLAN RSSI," Proc. EuroSSC, Oct 2007, Lake District, UK.

[9] I. Anderson and H. Muller, "Context awareness via GSM signal strength fluctuation," in Proc. Int. Conf. on Pervasive Computing, Late breaking results, Oesterreichische Computer Gesellschaft, May 2006, pp. 27-31.

[10] S. S. Chen, D. L. Donoho, and M. A. Saunders, "Atomic decomposition by basis pursuit," SIAM J. Scientific Computing, vol. 20, pp. 33-61, 1999.

[11] A. Antoniou and W.-S. Lu, Optimization: Methods, Algorithms, and Applications, Kluwer Academic Publishers, 2005. 
a) Decomposition Result: without movement

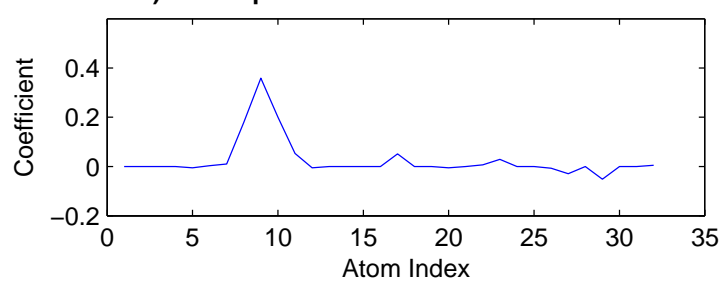

b) Decomposition Result: with movement

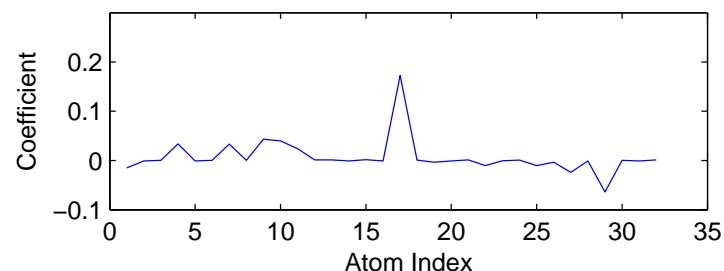

Fig. 3. The dictionary based decomposition results.

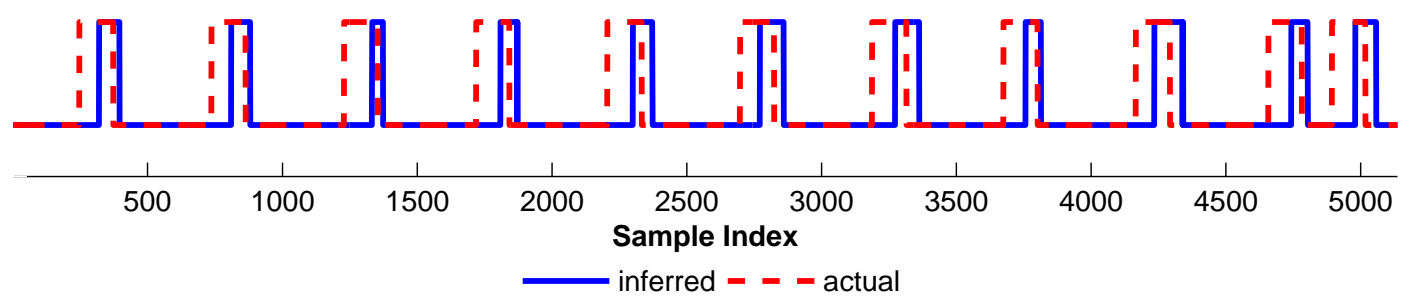

Fig. 4. Detection results for room $1(\mathrm{~N}=100)$.

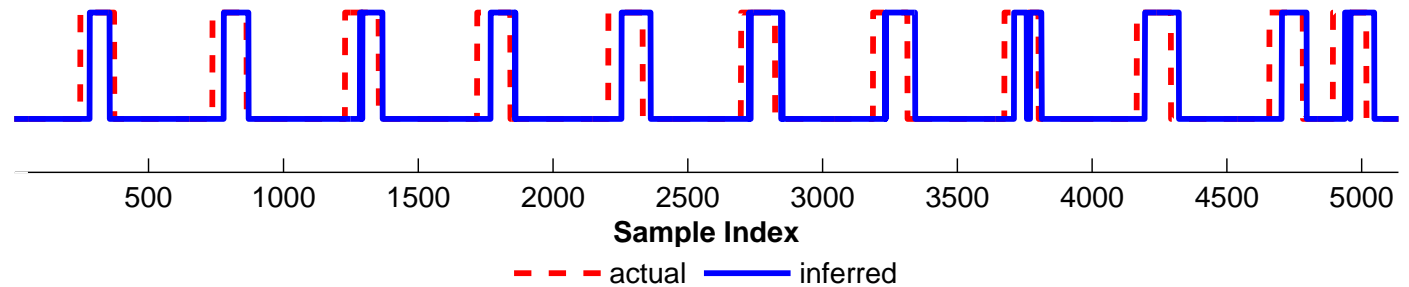

Fig. 5. Detection results for room $1(\mathrm{~N}=50)$.

a) RSSI fluctuation $(\mathrm{N}=50)$
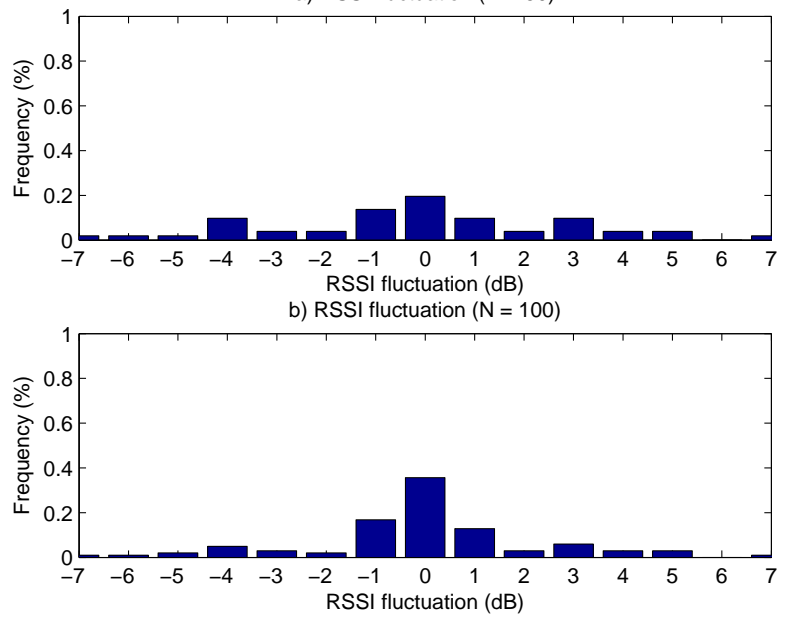

Fig. 6. RSSI fluctuations (sample index $=1300$ ).

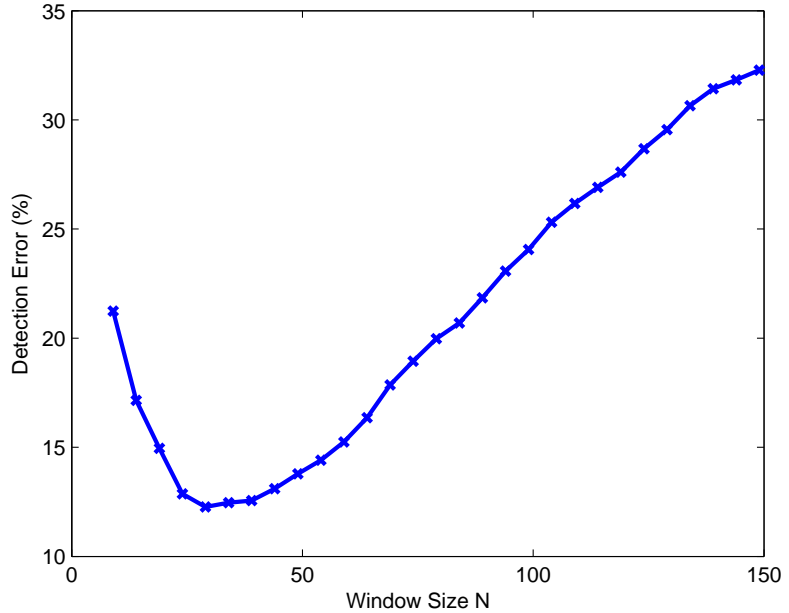

Fig. 7. Detection error rate for room 1. 


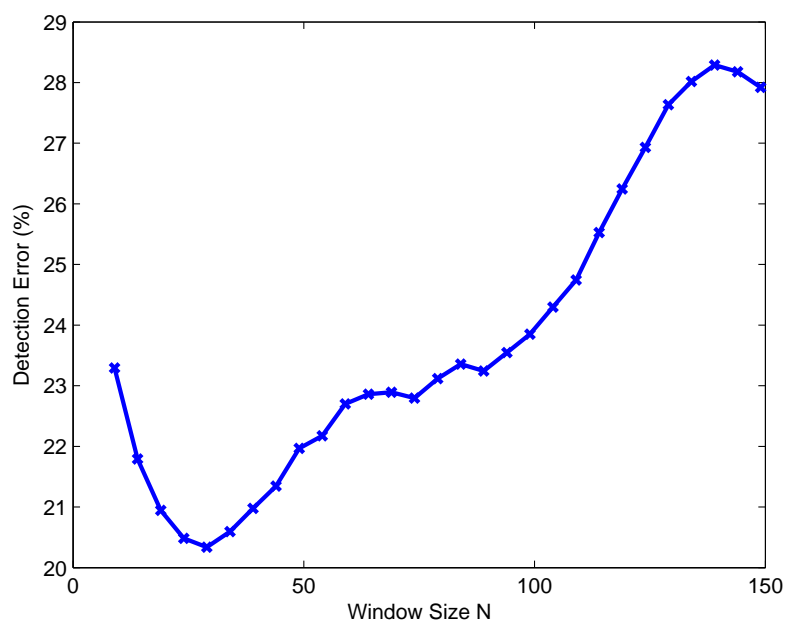

Fig. 8. Detection error rate for room 2.

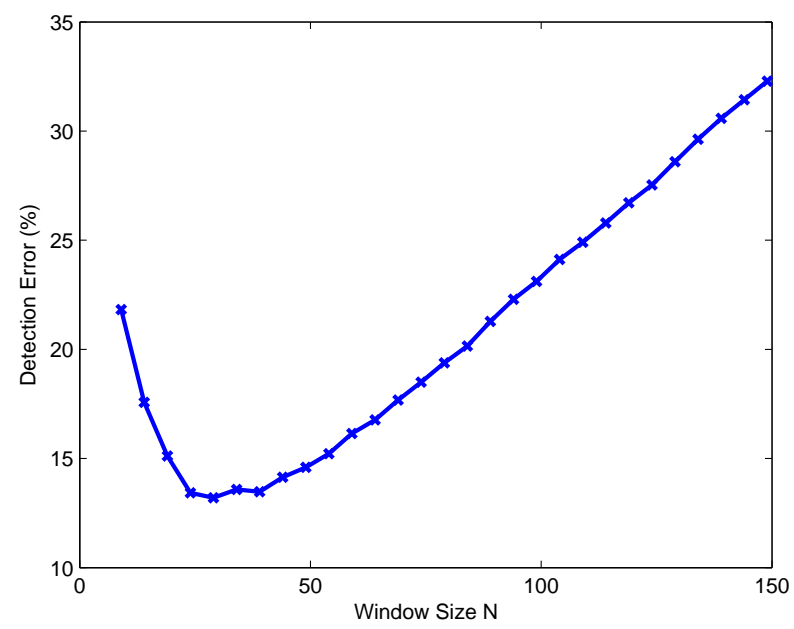

Fig. 9. Detection error rate for room 3.

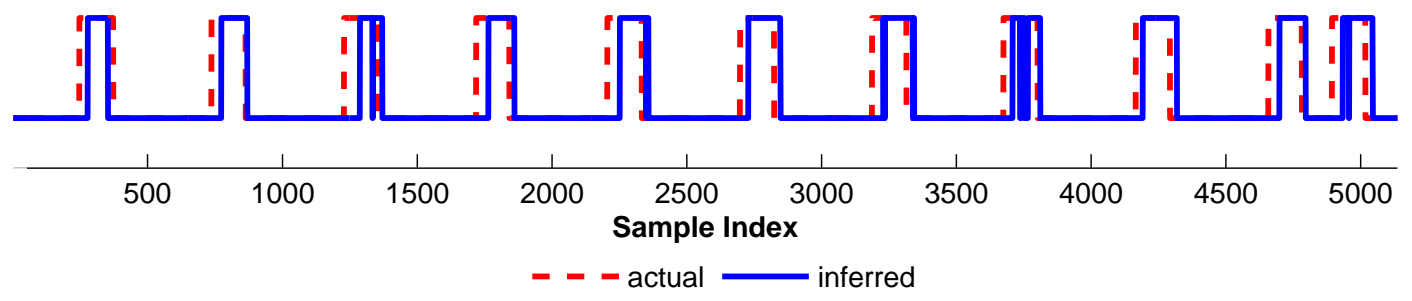

Fig. 10. Detection results for room $1(\mathrm{~N}=45)$.

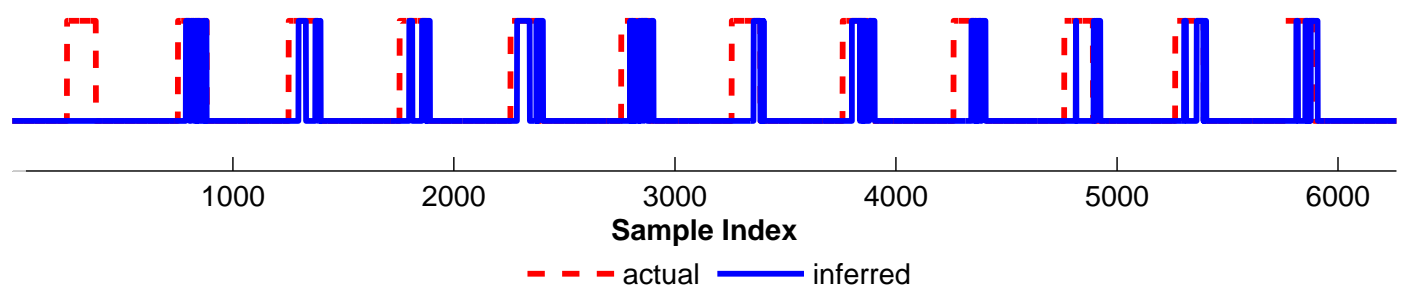

Fig. 11. Detection results for room $2(\mathrm{~N}=45)$.

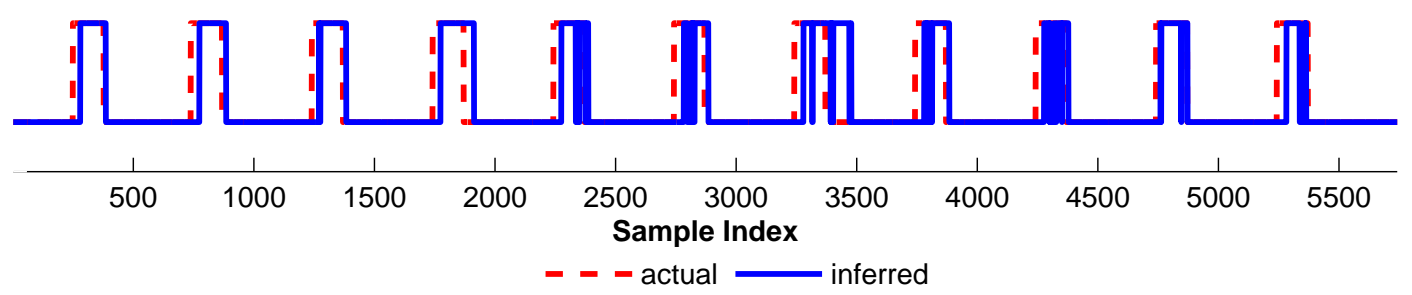

Fig. 12. Detection results for room $3(\mathrm{~N}=45)$. 